\title{
Novel Insight into the Role of Endoplasmic Reticulum Stress in the Pathogenesis of Myocardial Ischemia-Reperfusion Injury
}

\author{
Hang Zhu' ${ }^{1}$ and Hao Zhou ${ }^{10}{ }^{1,2}$ \\ ${ }^{1}$ Institute of Geriatric Cardiovascular Disease, Medical School of Chinese People's Liberation Army, China \\ ${ }^{2}$ Center for Cardiovascular Research and Alternative Medicine, University of Wyoming College of Health Sciences, USA \\ Correspondence should be addressed to Hao Zhou; zhouhao@plagh.org
}

Received 7 February 2021; Revised 28 February 2021; Accepted 17 March 2021; Published 28 March 2021

Academic Editor: Daniele Vergara

Copyright (c) 2021 Hang Zhu and Hao Zhou. This is an open access article distributed under the Creative Commons Attribution License, which permits unrestricted use, distribution, and reproduction in any medium, provided the original work is properly cited.

Impaired function of the endoplasmic reticulum (ER) is followed by evolutionarily conserved cell stress responses, which are employed by cells, including cardiomyocytes, to maintain and/or restore ER homeostasis. ER stress activates the unfolded protein response (UPR) to degrade and remove abnormal proteins from the ER lumen. Although the UPR is an intracellular defense mechanism to sustain cardiomyocyte viability and heart function, excessive activation initiates ER-dependent cardiomyocyte apoptosis. Myocardial ischemia/reperfusion $(\mathrm{I} / \mathrm{R})$ injury is a pathological process occurring during or after revascularization of ischemic myocardium. Several molecular mechanisms contribute to the pathogenesis of cardiac I/R injury. Due to the dual protective/degradative effects of ER stress on cardiomyocyte viability and function, it is of interest to understand the basic concepts, regulatory signals, and molecular processes involved in ER stress following myocardial I/R injury. In this review, therefore, we present recent findings related to the novel components of ER stress activation. The complex effects of ER stress and whether they mitigate or exacerbate myocardial I/R injury are summarized to serve as the basis for research into potential therapies for cardioprotection through control of ER homeostasis.

\section{Introduction}

Myocardial ischemia/reperfusion (I/R) injury occurs when myocardial tissues or cardiomyocytes are resupplied with fresh blood flow following a period of ischemia. In that situation, tissues/cells not only fail to recover from the ischemic damage but also develop additional injury caused by the reperfusion itself $[1,2]$. This phenomenon is particularly prominent in the heart, liver, and brain [3-5]. Clinically, cardiac surgery and coronary artery bypass graft may cause myocardial I/R injury [6-8]. It is now generally believed that the main mechanisms of reperfusion injury are excessive formation of free radicals within the tissue and intracellular calcium overload [9-11]. Among the various biochemical mechanisms and signal pathways that may be involved [12-14], endoplasmic reticulum (ER) stress has been found to be associated with reperfusion-mediated oxidative stress and cardiomyocyte death $[15,16]$. ER stress refers to a pathological process associated with hypoxia, starvation, calcium imbalance, and free radical overproduction that disrupts the physiological functions of the ER $[17,18]$. These stimuli may cause signaling from the ER to the cytoplasm and nucleus, where adaptive responses or the apoptotic program will be ultimately activated $[19,20]$.

Recent studies have reported a close relationship between ER stress and cardiac I/R injury $[21,22]$. This suggests reducing ER stress through genetic approaches or pharmacological treatments could potentially reduce myocardial $I / R$ injury [23-25], thereby bringing clinical benefits on many patients with cardiovascular disease. This review focuses on the current research investigating the role played by ER stress in myocardial $\mathrm{I} / \mathrm{R}$ injury with the aim of identifying clinical approaches that may be applied to reduce cardiac I/R injury in the future.

\section{Overview of Myocardial I/R Injury}

Nutrients are supplied to tissues and metabolic waste carried away by the circulation. Insufficient blood flow to a tissue, such as the myocardium, results in ischemia $[26,27]$, which 
can lead to cell death and tissue damage. Myocardial ischemia is usually caused by occlusion of one or more coronary arteries, which is followed by a decline in oxygen tension within the myocardium [2, 28, 29]. Myocardial ischemia severely hinders oxidative metabolism of fatty acids, glucose, pyruvate, and lactic acid, which causes energetic stress within cardiomyocytes $[30,31]$. It also slows or even stops mitochondrial respiration $[32,33]$, diminishing oxidative phosphorylation and ATP production. In the absence of sufficient oxygen, ATP production through glycolysis is enhanced, which leads to overproduction of lactic acid $[34,35]$ and, in turn, intracellular acidosis. In addition, ischemia interrupts $\beta$-oxidation of fatty acids and thus promotes accumulation of incomplete fatty acid metabolites in the cytoplasm $[36,37]$. The most important change within the ischemic myocardium is the reduced generation of high-energy phosphoric compounds (e.g., ATP) and cardiomyocyte death due to ATP deficiency $[38,39]$. As a result of the ATP undersupply, the calcium pump within cardiomyocytes cannot effectively remove calcium from the cytoplasm, resulting into calcium overload [40, 41]. The resultant abnormal calcium signal blunts ventricular contraction and promotes the development of cardiac dysfunction [42-44].

From the perspective of treatment, timely restoration of blood flow to the myocardium is an effective way to relieve tissue ischemia and insufficient nutrient supply $[45,46]$. Interestingly, however, reperfusion of ischemic tissue can cause additional damage due to $\mathrm{I} / \mathrm{R}$ injury $[47,48]$. This concept was first proposed in 1955 by Sewell et al., based on observations made in dogs after coronary artery ligation [49]. They reported that removing the coronary ligation, and thus restoring of myocardial perfusion, induced ventricular fibrillation and death $[50,51]$. This concept was further validated in 1960 by Jennings et al. [52], who reported that when tissue or cells regain a blood supply after transient ischemia, they undergo I/R injury. It was also shown that myocardial ischemia and subsequent reperfusion injury are independent but interrelated pathophysiological processes $[53,54]$. Consequently, the prevention and treatment of reperfusion injury should start during the ischemic period, and the ischemia must be removed as soon as possible $[55,56]$. The shorter the duration of ischemia, the smaller are the ischemic changes and the possibility of injury after reperfusion [57, 58]. At present, there is no particularly effective way to cope with myocardial I/R injury [59-61]. Several studies have been conducted to understand the molecular mechanisms underlying myocardial I/R injury. Oxidative stress, microvascular damage, inflammatory responses, autophagy inhibition, immune disorders, platelet activation, cardiomyocyte metabolic disturbance, ER stress, and mitochondrial dysfunction are all reported to be potential pathological factors contributing to the development of cardiac I/R injury [62-66].

\section{Molecular Basis of ER Stress}

3.1. Overview of the ER. The ER is a membranous tubular organelle within eukaryotic cells [67]. It is found in two forms: rough and smooth [68]. Rough ER localizes with ribosomes and is mainly responsible for protein folding and posttranslational modification $[69,70]$. Smooth ER, on the other hand, functions to maintain lipid biosynthesis and calcium storage [71]. ER stress is a state in which an external stimulus disrupts ER homeostasis and triggers the accumulation of unfolded or misfolded proteins within the ER lumen [72]. Calcium overload and abnormal lipid metabolism, due to ER dysregulation, will further promote ER stress [73]. The stimuli thought to cause ER stress include nutritional deficiency, hypoxia, ischemia, oxidative stress, and DNA damage [74-76]. When ER stress occurs, the cell reduces protein synthesis and promotes degradation of misfolded proteins [77]. However, under continuous strong stimulation, excessive ER stress is associated with cell apoptosis [78].

3.2. Activation of ER Stress. ER stress in mammals has four components: inhibition of protein translation, upregulation of molecular chaperones, activation of the protein degradative program, and induction of apoptosis [79]. ER stress signal transduction is mediated via three crucial enzymes (Figure 1) [80]: protein kinase R-link ER kinase (PERL), activating transcription factor-6 (ATF-6), and inositol-requiring enzyme-1 (IRE1). ER molecular chaperones acting as sensors of ER homeostasis play a key role in monitoring the accumulation of unfolded proteins within the ER [81]. Under physiological conditions, GRP78 (also known as binding immunoglobulin protein; BiP) binds to PERK, ATF-6, and IRE1 [82] within the ER. However, GRP78 has greater affinity for unfolded proteins; consequently, when ER homeostasis is disrupted, leading to accumulation of unfolded proteins within the ER, GRP78 dissociates from PERK, ATF-6, and IRE1, which results in the activation of ER stress signaling transduction pathways [83].

\subsection{The Transduction Pathways of ER Stress}

3.3.1. PERK Pathway. PERK is a transmembrane protein in the ER membrane [84]. After dissociation of GRP78, it forms a homodimer and is then activated by autophosphorylation. Phosphorylated PERK catalyzes the phosphorylation of eukaryotic initiation factor- $2 \alpha(\mathrm{elF} 2 \alpha)$ [85], which inactivates eIF $2 \alpha$-mediated translation. This effect significantly represses the transcription of most mRNA and, in turn, protein synthesis, which reduces the protein load on the ER [86]. Interestingly, elF $2 \alpha$ phosphorylation is associated with an increase in the transcription of activating transcription factor-4 (ATF-4), which, after translation, translocates the cell nucleus and functions to upregulate ER molecular chaperones [87]. However, if ER homeostasis cannot be restored, the continuous overexpression of ATF-4 will promote the upregulation of $\mathrm{C} / \mathrm{EBP}$ homologous protein (CHOP), a potential proapoptotic protein regulating cell death [88].

3.3.2. ATF-6 Pathway. Like PERK, ATF-6 is an ER transmembrane protein [89]. After dissociation of GRP78, ATF-6 translocates to the Golgi apparatus where it is cleaved and activated by the proteases Sit-1/2. The activated ATF- 6 migrates into the nucleus where it forms homodimers or 


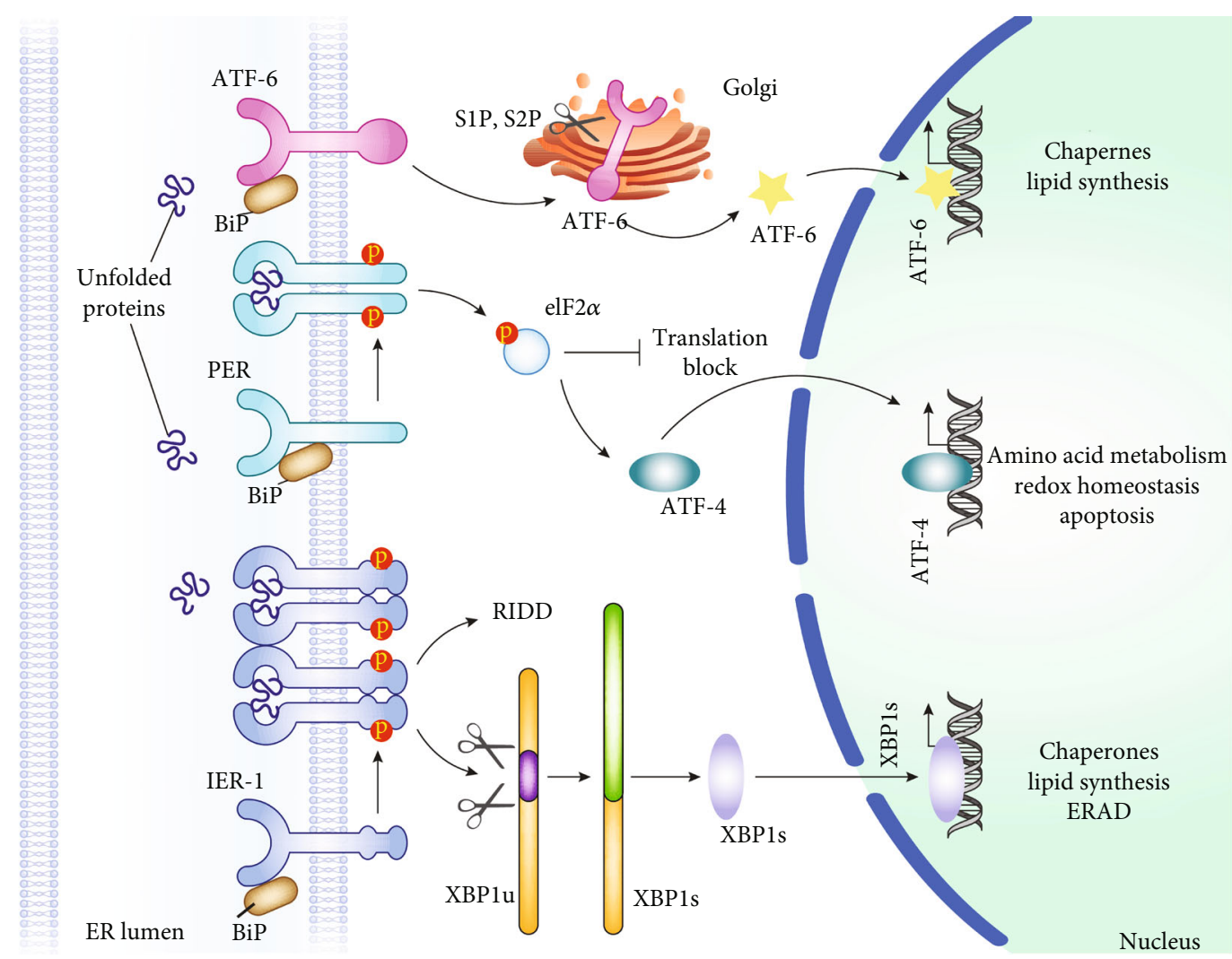

FIgURE 1: The regulatory mechanisms of endoplasmic reticulum (ER) stress. ER stress in mammals has four components: inhibition of protein translation, upregulation of molecular chaperones, activation of the protein degradative program, and induction of apoptosis [79]. ER stress signal transduction is mediated via three crucial enzymes [80]: protein kinase R-link ER kinase (PERL), activating transcription factor-6 (ATF-6), and inositol-requiring enzyme-1 (IRE1). ER molecular chaperones acting as sensors of ER homeostasis play a key role in monitoring the accumulation of unfolded proteins within the ER.

heterodimers with other transcription factors, leading to the upregulation of ER chaperone genes [90].

3.3.3. IRE1 Pathway. Upon dissociation of GRP78, the ER transmembrane protein IRE1 forms a homodimer and undergoes autophosphorylation activation [91]. Activated IRE1 has endoribonuclease activity [92], which can cut the mRNA encoding XBP1 ( $\mathrm{x}$-box binding protein-1) to form a new transcript encoding a second XBP1 isoform [93]. When abundant, the translated XPB1 protein migrates into the nucleus, where it upregulates the expression of genes related to ER stress [94]. Long-term activation of the IRE1 is associated with apoptosis activated via the TRAF2/ASK1/JNK pathway.

3.4. Unfolded Protein Response. After synthesis on the ribosomes, proteins must be folded and packaged correctly within the ER. Protein folding is carried out under redox conditions and requires two ER stress reactive proteins [95]: ER stress oxidoreductase (ERO) and disulfide proteolytic enzyme. After dissociation of GRP78 and their autoactivation (as described above), IRE1, ATF-6, and PERK respond to the presence of incorrectly folded proteins associated with ER stress [96, 97]. This is called the "unfolded protein response" (UPR) [98]. A key function of the ER is identification, control, and correction of protein quality. Proteins that cannot be folded correctly will be transferred from ER to the cytoplasm for degradation by the $26 \mathrm{~S}$ proteasome [99]. The early stage of the URP is the activation of proteasomeinduced degradation of unfolded proteins and the upregulation of XBP1 and ATF-4 [100]. These alterations are aimed at reducing the load of unfolded or misfolded proteins within ER. Later, an inflammatory response is activated via NF- $\kappa \mathrm{B}$ and JNK [101], which enhances defensive responses within the cytoplasm. If these responses are unable to restore ER function or cell homeostasis, the cell apoptosis program will be activated as the final stage of the UPR.

\section{Role of ER Stress in Myocardial I/R Injury}

4.1. ER Stress and Calcium Overload. Myocardial contraction relies on the oscillation of cytoplasmic free calcium concentration. Within cardiomyocytes, smooth ER (termed sarcoplasmic reticulum; SR) contains the primary calcium store. Excessive calcium release from SR into the cytoplasm leads to intracellular calcium overload, which is closely associated with cardiomyocyte contraction dysfunction and cell death [102-104]. ER dysfunction-mediated calcium overload plays an important role in myocardial I/R injury. During reperfusion, the function of the sodium-calcium exchanger and L-type calcium channels is impaired as a result of the insufficient oxygen supply during the ischemia [105]. By 


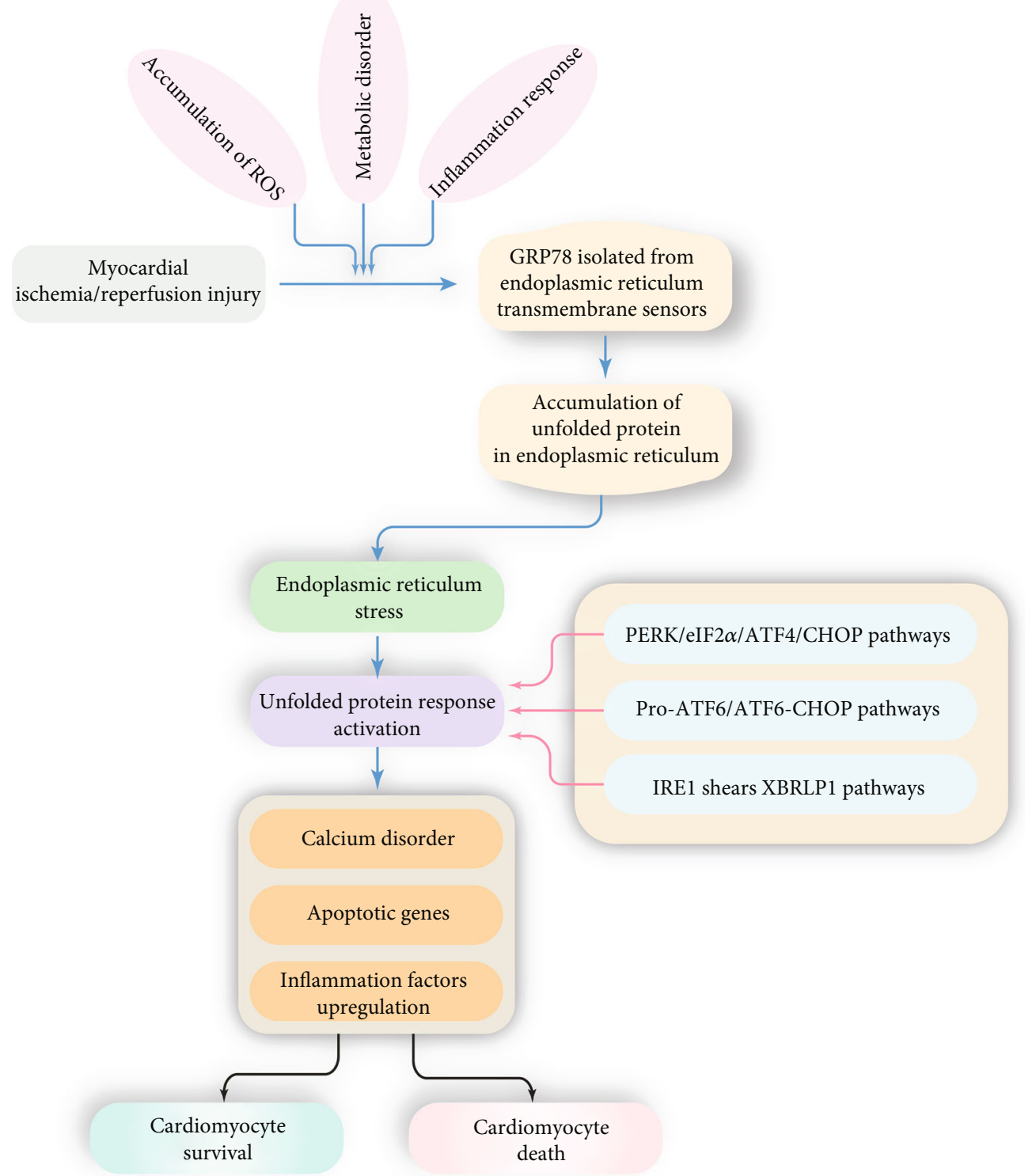

FIgURE 2: Role of endoplasmic reticulum (ER) stress in myocardial ischemia/reperfusion injury. ER stress is activated by accumulation of ROS, metabolic disorder, or inflammation response, which is featured by GRP78 isolation from ER. Then, unfolded protein accumulation in ER will activate the unfolded protein response (UPR) which is followed by calcium disorder, apoptotic gene upregulation, and inflammation response, resulting into cardiomyocyte death or survival dependent on the extent of ER stress.

contrast, the expression of calcium-sensitive receptors, such as 1,4,5-inositol trisphosphate receptor (IP3R), is significantly increased due to hypoxic stress or upregulation of hypoxia-inducible factor-1 (HIF1) [106]. These effects enhance calcium release from SR into the cytoplasm under conditions where physiological extrusion of calcium from the cell is suppressed. Thus, ER stress is an upstream trigger of cardiomyocyte calcium overload.

4.2. ER Stress and Cardiomyocyte Apoptosis. Once UPR fails to control the level of unfolded or misfolded proteins, ER stress will trigger the activation of apoptotic signaling. It is currently believed that ER stress can cause cardiomyocyte apoptosis via three pathways (Figure 2).
4.2.1. CHOP Pathway. CHOP is a transcription factor belonging to the C/EBP family. Under normal circumstances, $\mathrm{CHOP}$ expression is very low. The transcription and translation of CHOP are primarily regulated by IRE $1 \alpha$, ATF- 6 , and PERK $[107,108]$, and CHOP plays a key role in ER-induced apoptosis, such as that induced by I/R injury [109]. Upregulation of $\mathrm{CHOP}$ induces the expression of a variety of downstream proapoptotic and antiapoptotic genes, including Bcl-2, Bax, Bim, growth arrest and DNA damage-inducible protein 34 (GADD34), ER oxidoreductase-1 $\alpha$ (ERO1 $\alpha$ ), and the death receptor 5 (DR5) [110]. Among those, GADD34 promotes the expression of protein phosphatase1 (PP1), which in turn augments transcription of genes related to UPR [111]; ERO1 $\alpha$ triggers calcium leakage from 
the ER through IP3Rs, which leads to calcium overloaddependent cell apoptosis $[112,113]$; and DR5 triggers apoptosis through activation of caspase-8 [114].

4.2.2. IRE1 $\alpha / J N K$ Pathway. IRE $1 \alpha$ is a component of the most conserved pathway in mammalian UPR [115]. It has two active enzyme domains: a serine/threonine kinase domain and an endoribose nuclease (RNase) domain. When ER stress is induced, unfolded or misfolded proteins in the ER lumen directly bind to and activate IRE1 $\alpha$. Once activated, IRE $1 \alpha$ recruits tumor necrosis factor receptor-related factor-2 (TRAF2) and apoptotic-signaling kinase-1 (ASK1) [116], after which JNK is phosphorylated by the resultant IRE1 $\alpha$-TRAF2-ASK1 signaling complex $[117,118]$. Following cardiac I/R injury, activated JNK may promote cardiomyocyte apoptosis through phosphorylation of various members of the Bcl-2 family $[119,120]$. For example, JNK catalyzes phosphorylation of the antiapoptotic protein $\mathrm{Bcl}$ 2, which impairs its activity. At the same time JNK catalyzed, phosphorylation enhances the proapoptotic activity of Bim [121]. These alterations work together to mediate apoptosis in cardiomyocytes.

4.2.3. The Caspase-12 Pathway. The caspase-12 pathway is considered to be an ER-specific, nonmitochondrialdependent apoptotic pathway [122]. Caspase-12 activation is also a feature of ER stress-mediated cardiomyocyte apoptosis [123]. Under normal circumstances, caspase-12 binds to the ER membrane and forms a complex with TRAF2. ER stress directly induces caspase-12 dissociation from the ER membrane, enabling it to be activated by calpain $[124,125]$ or the IRE1 $\alpha$-TRAF2 complex [126]. Once activated, caspase-12 cleaves and activates caspase-9, which in turn cleaves and activates caspase- 3 to promote apoptosis [127].

\section{Summary and Outlook}

ER stress arises via multiple signaling pathways, expression of multiple genes, and participation of multiple stress factors. In cases of mild or early myocardial injury, ER stress involves a variety of protective proteins, which reduce the pathological stress on cardiomyocytes. However, excessive ER stress is associated with protein quality control disorder, resulting in the upregulation of apoptotic proteins. Notably, the role of ER stress during ischemia differs from that during reperfusion. It remains unclear whether ER stress is protective in the ischemic heart and only becomes lethal following reperfusion. In addition, although the molecular mechanisms underlying ER stress and its role in I/R injury have been characterized, the interactive effects of ER stress and other pathological alterations that occur during cardiac I/R injury, such as oxidative stress and mitochondrial dysfunction, are still not fully understood. Moreover, there are still no specific drugs targeting ER stress available in clinical practice. Additional investigations are therefore required to help us better understand the role of ER stress in myocardial $\mathrm{I} / \mathrm{R}$ injury.

\section{Data Availability}

All data generated or analyzed during this study are included in this published article.

\section{Conflicts of Interest}

All the authors declare that they have no conflicts of interest.

\section{References}

[1] G. Heusch, "Coronary microvascular obstruction: the new frontier in cardioprotection," Basic Research in Cardiology, vol. 114, no. 6, p. 45, 2019.

[2] J. Wang and H. Zhou, "Mitochondrial quality control mechanisms as molecular targets in cardiac ischemia $* *-* *$ reperfusion injury," Acta Pharmaceutica Sinica B, vol. 10, no. 10, pp. 1866-1879, 2020.

[3] J. Oliva, "Proteasome and organs ischemia-reperfusion injury," International Journal of Molecular Sciences, vol. 19, no. 1, p. 106, 2018.

[4] H. Zhou, J. Wang, P. Zhu et al., "NR4A1 aggravates the cardiac microvascular ischemia reperfusion injury through suppressing FUNDC1-mediated mitophagy and promoting Mff-required mitochondrial fission by CK2 $\alpha$," Basic Research in Cardiology, vol. 113, no. 4, p. 23, 2018.

[5] J. Wang, P. Zhu, S. Toan, R. Li, J. Ren, and H. Zhou, "Pum2Mff axis fine-tunes mitochondrial quality control in acute ischemic kidney injury," Cell Biology and Toxicology, vol. 36, no. 4, pp. 365-378, 2020.

[6] M. Aghaei, M. Motallebnezhad, S. Ghorghanlu et al., "Targeting autophagy in cardiac ischemia/reperfusion injury: a novel therapeutic strategy," Journal of Cellular Physiology, vol. 234, no. 10, pp. 16768-16778, 2019.

[7] Z. J. Mao, H. Lin, F. Y. Xiao, Z. Q. Huang, and Y. H. Chen, "Melatonin against myocardial ischemia-reperfusion injury: a meta-analysis and mechanism insight from animal studies," Oxidative Medicine and Cellular Longevity, vol. 2020, Article ID 1241065, 11 pages, 2020.

[8] T. Carbonell and A. V. Gomes, "MicroRNAs in the regulation of cellular redox status and its implications in myocardial ischemia-reperfusion injury," Redox Biology, vol. 36, article 101607, 2020.

[9] N. B. Liu, M. Wu, C. Chen et al., "Novel molecular targets participating in myocardial ischemia-reperfusion injury and cardioprotection," Cardiology Research and Practice, vol. 2019, Article ID 6935147, 16 pages, 2019.

[10] K. Boengler, J. Bornbaum, K. D. Schlüter, and R. Schulz, "P66shc and its role in ischemic cardiovascular diseases," Basic Research in Cardiology, vol. 114, no. 4, p. 29, 2019.

[11] S. An, X. Wang, H. Shi et al., "Apelin protects against ischemia-reperfusion injury in diabetic myocardium via inhibiting apoptosis and oxidative stress through PI3K and p38MAPK signaling pathways," Aging (Albany NY), vol. 12, no. 24, pp. 25120-25137, 2020.

[12] E. Chen, C. Chen, Z. Niu et al., "Poly(I:C) preconditioning protects the heart against myocardial ischemia/reperfusion injury through TLR3/PI3K/Akt-dependent pathway," Signal Transduction and Targeted Therapy, vol. 5, no. 1, p. 216, 2020.

[13] L. Zhang, S. Cai, S. Cao et al., "Diazoxide protects against myocardial ischemia/reperfusion injury by moderating ERS 
via regulation of the miR-10a/IRE1 pathway," Oxidative Medicine and Cellular Longevity, vol. 2020, Article ID 4957238, 16 pages, 2020.

[14] Y. Tan, D. Mui, S. Toan, P. Zhu, R. Li, and H. Zhou, "SERCA overexpression improves mitochondrial quality control and attenuates cardiac microvascular ischemia-reperfusion injury," Mol Ther Nucleic Acids, vol. 22, pp. 696-707, 2020.

[15] D. Han, Y. Wang, J. Chen et al., “Activation of melatonin receptor 2 but not melatonin receptor 1 mediates melatoninconferred cardioprotection against myocardial ischemia/reperfusion injury," Journal of Pineal Research, vol. 67, article e12571, 2019.

[16] L. M. Yu, X. Dong, J. Zhang et al., "Naringenin attenuates myocardial ischemia-reperfusion injury via cGMP-PKGI $\alpha$ signaling and in vivo and in vitro studies," Oxidative Medicine and Cellular Longevity, vol. 2019, Article ID 7670854, 15 pages, 2019.

[17] J. Sandoval, D. J. Orlicky, A. Allawzi et al., "Toxic acetaminophen exposure induces distal lung ER stress, proinflammatory signaling, and emphysematous changes in the adult murine lung," Oxidative Medicine and Cellular Longevity, vol. 2019, Article ID 7595126, 15 pages, 2019.

[18] Z. W. Chen, C. Y. Huang, J. F. Cheng, S. Y. Chen, L. Y. Lin, and C. K. Wu, "Stress echocardiography-derived E/e' predicts abnormal exercise hemodynamics in heart failure with preserved ejection fraction," Frontiers in Physiology, vol. 10, p. 1470, 2019.

[19] O. Moltedo, P. Remondelli, and G. Amodio, "The mitochondria-endoplasmic reticulum contacts and their critical role in aging and age-associated diseases," Frontiers in Cell and Development Biology, vol. 7, p. 172, 2019.

[20] J. Zhong, Y. Tan, J. Lu et al., "Therapeutic contribution of melatonin to the treatment of septic cardiomyopathy: a novel mechanism linking Ripk3-modified mitochondrial performance and endoplasmic reticulum function," Redox Biology, vol. 26, p. 101287, 2019.

[21] X. Zhang, C. S. Gibhardt, T. Will et al., "Redox signals at the ER-mitochondria interface control melanoma progression," The EMBO Journal, vol. 38, no. 15, article e100871, 2019.

[22] R. C. Yue, S. Z. Lu, Y. Luo et al., "Calpain silencing alleviates myocardial ischemia-reperfusion injury through the NLRP3/ ASC/Caspase-1 axis in mice," Life Sciences, vol. 233, p. 116631, 2019.

[23] S. Wang, W. Bian, J. Zhen, L. Zhao, and W. Chen, "Melatonin-mediated Pak2 activation reduces cardiomyocyte death through suppressing hypoxia reoxygenation injury-induced endoplasmic reticulum stress," Journal of Cardiovascular Pharmacology, vol. 74, no. 1, pp. 20-29, 2019.

[24] D. Shen, R. Chen, L. Zhang et al., "Sulodexide attenuates endoplasmic reticulum stress induced by myocardial ischaemia/reperfusion by activating the PI3K/Akt pathway," Journal of Cellular and Molecular Medicine, vol. 23, no. 8, pp. 5063-5075, 2019.

[25] G. Zhang, X. Wang, T. G. Gillette, Y. Deng, and Z. V. Wang, "Unfolded protein response as a therapeutic target in cardiovascular disease," Current Topics in Medicinal Chemistry, vol. 19, no. 21, pp. 1902-1917, 2019.

[26] J. Wang, S. Toan, and H. Zhou, "Mitochondrial quality control in cardiac microvascular ischemia-reperfusion injury: new insights into the mechanisms and therapeutic potentials," Pharmacological Research, vol. 156, p. 104771, 2020.
[27] J. Wang, S. Toan, and H. Zhou, "New insights into the role of mitochondria in cardiac microvascular ischemia/reperfusion injury," Angiogenesis, vol. 23, no. 3, pp. 299-314, 2020.

[28] J. Wang, P. Zhu, R. Li, J. Ren, Y. Zhang, and H. Zhou, "Bax inhibitor 1 preserves mitochondrial homeostasis in acute kidney injury through promoting mitochondrial retention of PHB2," Theranostics, vol. 10, no. 1, pp. 384-397, 2020.

[29] H. Zhou, P. Zhu, J. Wang, H. Zhu, J. Ren, and Y. Chen, "Pathogenesis of cardiac ischemia reperfusion injury is associated with CK2 $\alpha$-disturbed mitochondrial homeostasis via suppression of FUNDC1-related mitophagy," Cell Death and Differentiation, vol. 25, no. 6, pp. 1080-1093, 2018.

[30] W. E. Hughes, A. M. Beyer, and D. D. Gutterman, "Vascular autophagy in health and disease," Basic Research in Cardiology, vol. 115, no. 4, p. 41, 2020.

[31] P. Kleinbongard, "Cardioprotection by early metoprololattenuation of ischemic vs. reperfusion injury?" Basic Research in Cardiology, vol. 115, no. 5, p. 54, 2020.

[32] X. Yang, N. An, C. Zhong et al., "Enhanced cardiomyocyte reactive oxygen species signaling promotes ibrutinibinduced atrial fibrillation," Redox Biology, vol. 30, article 101432, 2020.

[33] M. Matzinger, K. Fischhuber, D. Pölöske, K. Mechtler, and E. H. Heiss, "AMPK leads to phosphorylation of the transcription factor Nrf2, tuning transactivation of selected target genes," Redox Biology, vol. 29, p. 101393, 2020.

[34] M. Manevski, T. Muthumalage, D. Devadoss et al., "Cellular stress responses and dysfunctional mitochondrial-cellular senescence, and therapeutics in chronic respiratory diseases," Redox Biology, vol. 33, p. 101443, 2020.

[35] F. Cao, M. L. Maguire, D. J. McAndrew et al., "Overexpression of mitochondrial creatine kinase preserves cardiac energetics without ameliorating murine chronic heart failure," Basic Research in Cardiology, vol. 115, no. 2, p. 12, 2020.

[36] H. Li and N. Xia, "The role of oxidative stress in cardiovascular disease caused by social isolation and loneliness," Redox Biology, vol. 37, article 101585, 2020.

[37] H. Jiang, D. Jia, B. Zhang et al., "Exercise improves cardiac function and glucose metabolism in mice with experimental myocardial infarction through inhibiting HDAC4 and upregulating GLUT1 expression," Basic Research in Cardiology, vol. 115, no. 3, p. 28, 2020.

[38] A. J. Causer, J. K. Shute, M. H. Cummings et al., "Circulating biomarkers of antioxidant status and oxidative stress in people with cystic fibrosis: a systematic review and meta-analysis," Redox Biology, vol. 32, p. 101436, 2020.

[39] A. R. Ednie and E. S. Bennett, "Intracellular O-linked glycosylation directly regulates cardiomyocyte L-type Ca2+ channel activity and excitation-contraction coupling," Basic Research in Cardiology, vol. 115, no. 6, p. 59, 2020.

[40] Y. Zhang, H. Zhou, W. Wu et al., "Liraglutide protects cardiac microvascular endothelial cells against hypoxia/reoxygenation injury through the suppression of the SR- $\mathrm{Ca}^{2+}-\mathrm{XO}-$ ROS axis via activation of the GLP-1R/PI3K/Akt/survivin pathways," Free Radical Biology \& Medicine, vol. 95, pp. 278-292, 2016.

[41] F. E. Mason, J. R. D. Pronto, K. Alhussini, C. Maack, and N. Voigt, "Cellular and mitochondrial mechanisms of atrial fibrillation," Basic Research in Cardiology, vol. 115, no. 6, p. 72,2020 . 
[42] H. Zhou, J. Wang, P. Zhu, S. Hu, and J. Ren, "Ripk3 regulates cardiac microvascular reperfusion injury: The role of IP3Rdependent calcium overload, XO-mediated oxidative stress and F-action/filopodia-based cellular migration," Cellular Signalling, vol. 45, pp. 12-22, 2018.

[43] K. Éva Sikura, Z. Combi, L. Potor et al., "Hydrogen sulfide inhibits aortic valve calcification in heart via regulating RUNX2 by NF- $\kappa$ B, a link between inflammation and mineralization," Journal of Advanced Research, vol. 27, pp. 165176, 2021.

[44] M. Dia, L. Gomez, H. Thibault et al., "Reduced reticulummitochondria $\mathrm{Ca} 2+$ transfer is an early and reversible trigger of mitochondrial dysfunctions in diabetic cardiomyopathy," Basic Research in Cardiology, vol. 115, no. 6, p. 74, 2020.

[45] R. K. Adapala, A. K. Kanugula, S. Paruchuri, W. M. Chilian, and C. K. Thodeti, "TRPV4 deletion protects heart from myocardial infarction-induced adverse remodeling via modulation of cardiac fibroblast differentiation," Basic Research in Cardiology, vol. 115, no. 2, p. 14, 2020.

[46] C. Maldonado, M. D. Nguyen, P. Bauer et al., "Rapid lipid modification of endothelial cell membranes in cardiac ische$\mathrm{mia} /$ reperfusion injury: a novel therapeutic strategy to reduce infarct size," Cardiovascular Drugs and Therapy, vol. 35, no. 1, pp. 113-123, 2021.

[47] Y. J. Zhang, M. Zhang, X. Zhao et al., "NAD(+) administration decreases microvascular damage following cardiac ischemia/reperfusion by restoring autophagic flux," Basic Research in Cardiology, vol. 115, no. 5, p. 57, 2020.

[48] M. V. Basalay, D. M. Yellon, and S. M. Davidson, "Targeting myocardial ischaemic injury in the absence of reperfusion," Basic Research in Cardiology, vol. 115, no. 6, p. 63, 2020.

[49] W. H. Sewell, D. R. Koth, and C. E. Huggins, "Ventricular fibrillation in dogs after sudden return of flow to the coronary artery," Surgery, vol. 38, no. 6, pp. 1050-1053, 1955.

[50] M. Kohlhauer, V. R. Pell, N. Burger et al., "Protection against cardiac ischemia-reperfusion injury by hypothermia and by inhibition of succinate accumulation and oxidation is additive," Basic Research in Cardiology, vol. 114, no. 3, p. 18, 2019.

[51] L. A. Bienvenu, A. Maluenda, J. D. McFadyen et al., "Combined antiplatelet/anticoagulant drug for cardiac ischemia/reperfusion injury," Circulation Research, vol. 127, no. 9, pp. 1211-1213, 2020.

[52] R. B. Jennings, H. M. Sommers, G. A. Smyth, H. A. Flack, and H. Linn, "Myocardial necrosis induced by temporary occlusion of a coronary artery in the dog," Archives of Pathology, vol. 70, pp. 68-78, 1960.

[53] Y. Guo, Y. Nong, D. N. Tukaye et al., "Inducible cardiacspecific overexpression of cyclooxygenase-2 (COX-2) confers resistance to ischemia/reperfusion injury," Basic Research in Cardiology, vol. 114, no. 5, p. 32, 2019.

[54] Y. Li, P. Liang, B. Jiang et al., "CARD9 promotes autophagy in cardiomyocytes in myocardial ischemia/reperfusion injury via interacting with Rubicon directly," Basic Research in Cardiology, vol. 115, no. 3, p. 29, 2020.

[55] X. M. Gao, Y. Su, S. Moore et al., "Relaxin mitigates microvascular damage and inflammation following cardiac ischemiareperfusion," Basic Research in Cardiology, vol. 114, no. 4, p. 30, 2019.

[56] Y. Sawashita, N. Hirata, Y. Yoshikawa, H. Terada, Y. Tokinaga, and M. Yamakage, "Remote ischemic preconditioning reduces myocardial ischemia-reperfusion injury through unacylated ghrelin-induced activation of the JAK/ STAT pathway," Basic Research in Cardiology, vol. 115, no. 4, p. 50, 2020.

[57] S. Lahnwong, S. Palee, N. Apaijai et al., "Acute dapagliflozin administration exerts cardioprotective effects in rats with cardiac ischemia/reperfusion injury," Cardiovascular Diabetology, vol. 19, no. 1, p. 91, 2020.

[58] M. Lobo-Gonzalez, C. Galán-Arriola, X. Rossello et al., "Metoprolol blunts the time-dependent progression of infarct size," Basic Research in Cardiology, vol. 115, no. 5, p. 55, 2020.

[59] Z. He, S. Davidson, and D. Yellon, "The importance of clinically relevant background therapy in cardioprotective studies," Basic Research in Cardiology, vol. 115, no. 6, p. 69, 2020.

[60] A. Clementi, G. M. Virzì, A. Brocca et al., "Advances in the pathogenesis of cardiorenal syndrome type 3," Oxidative Medicine and Cellular Longevity, vol. 2015, Article ID 148082, 8 pages, 2015.

[61] H. Bøtker, "The changing face after acute myocardial infarction," Basic Research in Cardiology, vol. 115, p. 5, 2020.

[62] B. Chan, A. Roczkowsky, W. J. Cho et al., "Junctophilin-2 is a target of matrix metalloproteinase-2 in myocardial ischemiareperfusion injury," Basic Research in Cardiology, vol. 114, no. 6, p. 42, 2019.

[63] Y. Yang, G. Yang, L. Yu et al., “An interplay between MRTFA and the histone acetyltransferase TIP60 mediates hypoxiareoxygenation induced iNOS transcription in macrophages," Frontiers in Cell and Development Biology, vol. 8, p. 484, 2020.

[64] M. Wallert, M. Ziegler, X. Wang et al., " $\alpha$-Tocopherol preserves cardiac function by reducing oxidative stress and inflammation in ischemia/reperfusion injury," Redox O Biologico, vol. 26, article 101292, 2019.

[65] C. Xiao, M. L. Xia, J. Wang et al., "Luteolin attenuates cardiac ischemia/reperfusion injury in diabetic rats by modulating Nrf2 antioxidative function," Oxidative Medicine and Cellular Longevity, vol. 2019, Article ID 2719252, 9 pages, 2019.

[66] T. Alakoski, J. Ulvila, R. Yrjölä et al., "Inhibition of cardiomyocyte Sproutyl protects from cardiac ischemia-reperfusion injury," Basic Research in Cardiology, vol. 114, no. 2, p. 7, 2019.

[67] E. Bovo, R. Nikolaienko, S. Bhayani et al., "Novel approach for quantification of endoplasmic reticulum $\mathrm{Ca}(2+)$ transport," American Journal of Physiology. Heart and Circulatory Physiology, vol. 316, no. 6, pp. H1323-h1331, 2019.

[68] Y. Ji, Y. Ge, X. Xu et al., "Vildagliptin reduces stenosis of injured carotid artery in diabetic mouse through inhibiting vascular smooth muscle cell proliferation via ER stress/NF«B pathway," Frontiers in Pharmacology, vol. 10, p. 142, 2019.

[69] J. Lin, P. Walter, and T. Yen, "Endoplasmic reticulum stress in disease pathogenesis," Annual Review of Pathology, vol. 3, no. 1, pp. 399-425, 2008.

[70] C. Xu, B. Bailly-Maitre, and J. Reed, "Endoplasmic reticulum stress: cell life and death decisions," The Journal of Clinical Investigation, vol. 115, no. 10, pp. 2656-2664, 2005.

[71] C. Adams, M. Kopp, N. Larburu, P. Nowak, and M. Ali, "Structure and molecular mechanism of ER stress signaling by the unfolded protein response signal activator IRE1," Frontiers in Molecular Biosciences, vol. 6, p. 11, 2019.

[72] H. Zhu, Q. Jin, Y. Li et al., "Melatonin protected cardiac microvascular endothelial cells against oxidative stress injury via suppression of IP3R-[Ca(2+)]c/VDAC- $[\mathrm{Ca}(2+)] \mathrm{m}$ axis by activation of MAPK/ERK signaling pathway," Cell Stress \& Chaperones, vol. 23, no. 1, pp. 101-113, 2018. 
[73] C. Costa, W. Manaa, E. Duplan, and F. Checler, "The endoplasmic reticulum stress/unfolded protein response and their contributions to Parkinson's disease physiopathology," Cells, vol. 9, no. 11, p. 2495, 2020.

[74] K. Meyerovich, F. Ortis, F. Allagnat, and A. Cardozo, "Endoplasmic reticulum stress and the unfolded protein response in pancreatic islet inflammation," Journal of Molecular Endocrinology, vol. 57, no. 1, pp. R1-R17, 2016.

[75] M. Kitamura, "Endoplasmic reticulum stress and unfolded protein response in renal pathophysiology: Janus faces," American Journal of Physiology. Renal Physiology, vol. 295, no. 2, pp. F323-F334, 2008.

[76] K. Bhattarai, M. Chaudhary, H. Kim, and H. Chae, "Endoplasmic reticulum (ER) stress response failure in diseases," Trends in Cell Biology, vol. 30, no. 9, pp. 672-675, 2020.

[77] H. Hemingway, A. Moore, A. Olivencia-Yurvati, and S. Romero, "Effect of endoplasmic reticulum stress on endothelial ischemia-reperfusion injury in humans," American journal of physiology. Regulatory, integrative and comparative physiology, vol. 319, no. 6, pp. R666-R672, 2020.

[78] J. Ayyappan, K. Lizardo, S. Wang, E. Yurkow, and J. Nagajyothi, "Inhibition of SREBP improves cardiac lipidopathy, improves endoplasmic reticulum stress, and modulates chronic Chagas cardiomyopathy," Journal of the American Heart Association, vol. 9, no. 3, article e014255, 2020.

[79] J. Hong, K. Kim, J. H. Kim, and Y. Park, “The role of endoplasmic reticulum stress in cardiovascular disease and exercise," International Journal of Vascular Medicine, vol. 2017, 9 pages, 2017.

[80] S. Wang, P. Binder, Q. Fang et al., "Endoplasmic reticulum stress in the heart: insights into mechanisms and drug targets," British Journal of Pharmacology, vol. 175, no. 8, pp. 1293-1304, 2018.

[81] M. L. Battson, D. M. Lee, and C. L. Gentile, "Endoplasmic reticulum stress and the development of endothelial dysfunction," American Journal of Physiology. Heart and Circulatory Physiology, vol. 312, no. 3, pp. H355-h367, 2017.

[82] A. Cimellaro, M. Perticone, T. V. Fiorentino, A. Sciacqua, and M. L. Hribal, "Role of endoplasmic reticulum stress in endothelial dysfunction," Nutrition, Metabolism, and Cardiovascular Diseases, vol. 26, no. 10, pp. 863-871, 2016.

[83] W. S. Lee, W. H. Yoo, and H. J. Chae, "ER stress and autophagy," Current Molecular Medicine, vol. 15, no. 8, pp. 735-745, 2015.

[84] L. Cominacini, C. Mozzini, U. Garbin et al., "Endoplasmic reticulum stress and Nrf2 signaling in cardiovascular diseases," Free Radical Biology \& Medicine, vol. 88, pp. 233242, 2015.

[85] E. Sozen, B. Karademir, and N. K. Ozer, "Basic mechanisms in endoplasmic reticulum stress and relation to cardiovascular diseases," Free Radical Biology \& Medicine, vol. 78, pp. 3041, 2015.

[86] X. Zhou, B. Lu, D. Fu, M. Gui, L. Yao, and J. Li, "Huoxue Qianyang decoction ameliorates cardiac remodeling in obese spontaneously hypertensive rats in association with ATF6$\mathrm{CHOP}$ endoplasmic reticulum stress signaling pathway regulation," Biomedicine \& Pharmacotherapy, vol. 121, p. 109518, 2020.

[87] J. Nie, N. Ta, L. Liu, G. Shi, T. Kang, and Z. Zheng, “Activation of CaMKII via ER-stress mediates coxsackievirus B3- induced cardiomyocyte apoptosis," Cell Biology International, vol. 44, no. 2, pp. 488-498, 2019.

[88] J. Zhang, L. Wang, W. Xie et al., "Melatonin attenuates ER stress and mitochondrial damage in septic cardiomyopathy: a new mechanism involving BAP31 upregulation and MAPK-ERK pathway," Journal of Cellular Physiology, vol. 235, no. 3, pp. 2847-2856, 2020.

[89] R. Lenin, P. G. Nagy, K. A. Jha, and R. Gangaraju, "GRP78 translocation to the cell surface and O-GlcNAcylation of VE-cadherin contribute to ER stress-mediated endothelial permeability," Scientific Reports, vol. 9, no. 1, p. 10783, 2019.

[90] M. di Somma, M. Vliora, E. Grillo et al., "Role of VEGFs in metabolic disorders," Angiogenesis, vol. 23, no. 2, pp. 119130, 2020.

[91] S. Mohan, M. R. Preetha Rani, L. Brown, P. Ayyappan, and K. G. Raghu, "Endoplasmic reticulum stress: a master regulator of metabolic syndrome," European Journal of Pharmacology, vol. 860, article 172553, 2019.

[92] P. Fournier, C. Viallard, A. Dejda, P. Sapieha, B. Larrivée, and I. Royal, "The protein tyrosine phosphatase PTPRJ/DEP-1 contributes to the regulation of the Notch-signaling pathway and sprouting angiogenesis," Angiogenesis, vol. 23, no. 2, pp. 145-157, 2020.

[93] M. Navas-Madroñal, C. Rodriguez, M. Kassan et al., "Enhanced endoplasmic reticulum and mitochondrial stress in abdominal aortic aneurysm," Clinical Science (London, England), vol. 133, no. 13, pp. 1421-1438, 2019.

[94] A. Domingues, C. Boisson-Vidal, P. Marquet de Rouge et al., "Targeting endothelial thioredoxin-interacting protein (TXNIP) protects from metabolic disorder-related impairment of vascular function and post-ischemic revascularisation," Angiogenesis, vol. 23, no. 2, pp. 249-264, 2020.

[95] J. I. Abe, K. A. Ko, S. Kotla et al., "MAGI1 as a link between endothelial activation and ER stress drives atherosclerosis," JCI Insight, vol. 4, no. 7, 2019.

[96] M. Thamsen, R. Ghosh, V. C. Auyeung et al., "Small molecule inhibition of IRE1 $\alpha$ kinase/RNase has anti-fibrotic effects in the lung," PLoS One, vol. 14, no. 1, article e0209824, 2019.

[97] X. Wu, L. Zhang, Y. Miao et al., "Homocysteine causes vascular endothelial dysfunction by disrupting endoplasmic reticulum redox homeostasis," Redox Biology, vol. 20, pp. 46-59, 2019.

[98] T. Ji, X. Zhang, Z. Xin et al., "Does perturbation in the mitochondrial protein folding pave the way for neurodegeneration diseases?," Ageing Research Reviews, vol. 57, p. 100997, 2020.

[99] O. M. Amen, S. D. Sarker, R. Ghildyal, and A. Arya, "Endoplasmic reticulum stress activates unfolded protein response signaling and mediates inflammation, obesity, and cardiac dysfunction: therapeutic and molecular approach," Frontiers in Pharmacology, vol. 10, p. 977, 2019.

[100] V. Tang, S. Fu, B. S. Rayner, and C. L. Hawkins, "8-Chloroadenosine induces apoptosis in human coronary artery endothelial cells through the activation of the unfolded protein response," Redox Biology, vol. 26, p. 101274, 2019.

[101] C. Hofmann, H. A. Katus, and S. Doroudgar, "Protein misfolding in cardiac disease," Circulation, vol. 139, no. 18, pp. 2085-2088, 2019.

[102] E. L. Wilson and E. Metzakopian, "ER-mitochondria contact sites in neurodegeneration: genetic screening approaches to 
investigate novel disease mechanisms," Cell Death and Differentiation, 2020.

[103] H. N. Cha, C. H. Woo, H. Y. Kim, and S. Y. Park, "Methionine sulfoxide reductase B3 deficiency inhibits the development of diet-induced insulin resistance in mice," Redox Biology, vol. 38, p. 101823, 2021.

[104] S. Sharma, P. Chaudhary, R. Sandhir et al., "Heat-induced endoplasmic reticulum stress in soleus and gastrocnemius muscles and differential response to UPR pathway in rats," Cell Stress \& Chaperones, vol. 26, no. 2, pp. 323-339, 2021.

[105] B. A. Niemeyer, "TRICking SOCE into altered oscillations," Cell Calcium, vol. 92, p. 102290, 2020.

[106] A. A. Mohsin, J. Thompson, Y. Hu, J. Hollander, E. J. Lesnefsky, and Q. Chen, "Endoplasmic reticulum stressinduced complex I defect: central role of calcium overload," Archives of Biochemistry and Biophysics, vol. 683, p. 108299, 2020.

[107] M. L. O’Byrne, J. Huang, I. Asztalos et al., "Pediatric/congenital cardiac catheterization quality: an analysis of existing metrics," JACC. Cardiovascular Interventions, vol. 13, no. 24, pp. 28532864, 2020.

[108] T. L. Capasso, B. Li, H. J. Volek et al., "BMP10-mediated ALK1 signaling is continuously required for vascular development and maintenance," Angiogenesis, vol. 23, no. 2, pp. 203-220, 2020.

[109] Y. Miyazaki, K. Kaikita, M. Endo et al., "C/EBP homologous protein deficiency attenuates myocardial reperfusion injury by inhibiting myocardial apoptosis and inflammation," Arteriosclerosis, Thrombosis, and Vascular Biology, vol. 31, no. 5, pp. 1124-1132, 2011.

[110] B. Dong, H. Zhou, C. Han et al., "Ischemia/reperfusioninduced $\mathrm{CHOP}$ expression promotes apoptosis and impairs renal function recovery: the role of acidosis and GPR4," PLoS One, vol. 9, no. 10, article e110944, 2014.

[111] X. F. Guo and X. J. Yang, "Endoplasmic reticulum stress response in spontaneously hypertensive rats is affected by myocardial ischemia reperfusion injury," Experimental and Therapeutic Medicine, vol. 9, no. 2, pp. 319-326, 2015.

[112] M. R. Noh, J. I. Kim, S. J. Han, T. J. Lee, and K. M. Park, "C/EBP homologous protein (CHOP) gene deficiency attenuates renal ischemia/reperfusion injury in mice," Biochimica et Biophysica Acta, vol. 1852, no. 9, pp. 1895-1901, 2015.

[113] J. Bai, M. Khajavi, L. Sui et al., "Angiogenic responses in a 3D micro-engineered environment of primary endothelial cells and pericytes," Angiogenesis, vol. 24, no. 1, pp. 111-127, 2021.

[114] M. Chen, Y. Y. Zheng, Y. T. Song et al., "Pretreatment with low-dose gadolinium chloride attenuates myocardial ischemia/reperfusion injury in rats," Acta Pharmacologica Sinica, vol. 37, no. 4, pp. 453-462, 2016.

[115] T. Wu, N. Jiang, Z. Ji, and G. Shi, "The IRE1 signaling pathway is involved in the protective effect of low-dose LPS on myocardial ischemia-reperfusion injury," Life Sciences, vol. 231, p. 116569, 2019.

[116] E. Sozen, B. Yazgan, O. E. Tok et al., "Cholesterol induced autophagy via IRE1/JNK pathway promotes autophagic cell death in heart tissue," Metabolism, vol. 106, p. 154205, 2020.

[117] H. Chen, H. Yang, L. Pan et al., "The molecular mechanisms of XBP-1 gene silencing on IRE1 $\alpha$-TRAF2-ASK1-JNK pathways in oral squamous cell carcinoma under endoplasmic reticulum stress," Biomedicine \& Pharmacotherapy, vol. 77, pp. 108-113, 2016.
[118] A. L. Bayliss, A. Sundararaman, C. Granet, and H. Mellor, "Raftlin is recruited by neuropilin-1 to the activated VEGFR2 complex to control proangiogenic signaling," Angiogenesis, vol. 23, no. 3, pp. 371-383, 2020.

[119] Q. Jin, R. Li, N. Hu et al., "DUSP1 alleviates cardiac ischemia/reperfusion injury by suppressing the Mff- required mitochondrial fission and Bnip3-related mitophagy via the JNK pathways," Redox Biology, vol. 14, pp. 576-587, 2018.

[120] W. Xu, L. Zhang, Y. Zhang, K. Zhang, Y. Wu, and D. Jin, "TRAF1 exacerbates myocardial ischemia reperfusion injury via ASK1-JNK/p38 signaling," Journal of the American Heart Association, vol. 8, no. 21, article e012575, 2019.

[121] H. H. Su, J. M. Liao, Y. H. Wang et al., "Exogenous GDF11 attenuates non-canonical TGF- $\beta$ signaling to protect the heart from acute myocardial ischemia-reperfusion injury," Basic Research in Cardiology, vol. 114, no. 3, p. 20, 2019.

[122] M. Liu, Y. Wang, Q. Zhu et al., "Protective effects of circulating microvesicles derived from ischemic preconditioning on myocardial ischemia/reperfusion injury in rats by inhibiting endoplasmic reticulum stress," Apoptosis, vol. 23, no. 7-8, pp. 436-448, 2018.

[123] J. G. Xia, F. F. Xu, Y. Qu, D. D. Song, H. Shen, and X. H. Liu, "Atorvastatin post-conditioning attenuates myocardial ischemia reperfusion injury via inhibiting endoplasmic reticulum stress-related apoptosis," Shock, vol. 42, no. 4, pp. 365-371, 2014.

[124] J. Guo, Y. Bian, R. Bai, H. Li, M. Fu, and C. Xiao, “Globular adiponectin attenuates myocardial ischemia/reperfusion injury by upregulating endoplasmic reticulum $\mathrm{Ca}^{2+}$-ATPase activity and inhibiting endoplasmic reticulum stress," Journal of Cardiovascular Pharmacology, vol. 62, no. 2, pp. 143-153, 2013.

[125] E. H. Moon, Y. H. Kim, P. N. Vu et al., "TMEM100 is a key factor for specification of lymphatic endothelial progenitors," Angiogenesis, vol. 23, no. 3, pp. 339-355, 2020.

[126] T. Yoneda, K. Imaizumi, K. Oono et al., "Activation of caspase-12, an endoplastic reticulum (ER) resident caspase, through tumor necrosis factor receptor-associated factor 2dependent mechanism in response to the ER stress," The Journal of Biological Chemistry, vol. 276, no. 17, pp. 1393513940, 2001.

[127] D. Datta, A. Singh, D. R. Saha et al., “_Mycobacterium fortuitum_ -induced ER-Mitochondrial calcium dynamics promotes calpain/caspase-12/caspase- 9 mediated apoptosis in fish macrophages," Cell Death Discovery, vol. 4, no. 1, p. 30, 2018. 